\title{
Composition of Atmospheres of Normal and Peculiar stars
}

\author{
Dr. Omar Alhawi ${ }^{1}$, Dr. Firas Mazyed ${ }^{2}$ \\ Head of Department, Math \& Science, College of Engineering, Manama, Bahrain ${ }^{1}$ \\ Faculty Member, Department of Physics, College of Science, Wasit, Iraq ${ }^{2}$
}

\begin{abstract}
Context. The study of the chemical composition of stellar atmospheres is the main and most valuable source of information to determine the fundamental characteristics of stellar atmospheres, in one hand, and to studying the evolution of matter in the interior of stars, in the interstellar medium, and matter of the Galaxy, on the other hand. Aims. This study is to confirm that the effects of gravitational diffusion and light pressure begin to work, starting from specific degree of temperatures atmospheres of the main succession stars. Methods. Within the framework of this study, we processed Echelle spectra of the star "99Her" which have a spectral classes A-F, obtained with a 1.5-meter telescope PTT-150. In the investigation in question strengths of large numbers of absorption lines in stellar spectra were determined through photographic photometry of high resolution spectra, and the line strengths were than evaluated and converted into relative abundances on the basis of theoretical calculations of physical properties of model stellar atmospheres. Results. We determined the fundamental parameters of atmospheres:-the effective temperature, the acceleration of gravity, the micro-turbulent velocity. We also calculated the content of the chemical elements.
\end{abstract}

Keywords: Star Abundances, Chemical Compositions, Normal Stars, Chemically Peculiar

\section{INTRODUCTION}

The study of the chemical composition of stars is important both in terms of determining the fundamental characteristics of stellar atmospheres, and in studying the evolution of stars and matter of the Galaxy. The number and relative content of chemical elements in stars and interstellar medium depend on many different processes. For example, the history of star formation, the exchange of mass with the surrounding galaxy environment, the form of the Initial Mass Function (NFM) and how the stars of different masses evolve. The study of the chemical composition of stellar atmospheres is, in fact, the main and most valuable source of information on the evolution of matter in the interior of stars, in the interstellar medium, and in the volume of the Galaxy as a whole. [1]

Theoretical models of the chemical evolution of the Galaxy try to explain the observed ratios of the contents of elements relative to iron, as well as the radial gradient of the contents of elements along the radius of the Galaxy. In addition to ours, other galaxies were formed from gas that did not contain heavy elements. During the Big Bang, only the lightest elements were synthesized, such as Helium, Lithium, and Beryllium. [2]

The synthesis of heavier nuclei occurred already inside the stars. Some of these nuclei were formed at fairly quiet stages of the evolution of the star, and they fell into the interstellar medium through intense mass loss (in the Asymptotic Branch of the Giants, for example). Other elements were synthesized and thrown into the surrounding space during supernova explosions. Thus, the interstellar medium was enriched with relatively heavy elements. The enriched substance served as the starting material for the formation of the next generation of stars, which, in turn, also synthesized metals and threw them into the interstellar medium. That is, there was a cycle of matter in the Galaxy, during which the gas periodically fell into the next star, where he changed his chemical composition. The process depended on the rate of star formation in various regions of the Galaxy, as well as on the interstellar gas density. [3]

\section{A-STARS AND EARLY F-STARS}

Stars of spectral class A and early subclasses F have an effective temperature in the range of $7000-10000 \mathrm{~K}$. Considering data on the chemical composition, several types can be distinguished here, including chemical peculiarities[3]:

1. "Normal" stars of class A.

2. Magnetic Ap stars.

3. Am-stars. 


\section{International Advanced Research Journal in Science, Engineering and Technology}

Vol. 6, Issue 3, March 2019

Let us consider each group separately.

"Normal" stars.

They are stars with solar chemical composition. For various reasons, such stars are difficult to indicate. For a long time, some stars were used as standards in the analysis of the chemical composition of other A-stars. Often, those stars that were believed to have a normal chemical composition later turned out to be of type Am. Quite often the only exact criterion for belonging to this type was the excess of heavy elements. Am stars do not differ from normal stars in the sense that they usually lack a magnetic field and variability of brightness. The content of relatively light metals is often close to solar. But only with careful analysis of the spectral lines of heavier metals, it can be established that the star is of type Am. A typical example is the Sirius star, previously classified as normal.

You can also specify Vega. Studies have shown that its composition is different from solar (it has a general deficit of metals). Vega refers to the class of luminosity V and, possibly, to stars of the type Lambda Boo, surrounded by a dust envelope, masking the real chemical composition of the star. Reduced metallicity in the spectral class A can be observed not only in dwarfs, but also in giants. For example, HR7338 ( $\alpha$ Dra), belonging to the class A0 III. In A-super giants, the content of heavy elements does not show large differences from solar elements. Perhaps small deviations from the normal chemical composition among super giants in the vicinity of the Sun reflect the corresponding in homogeneities in the chemical composition of the interstellar medium. It is also likely that the parameters Teff and logg could be inaccurately determined. [4]

In a number of cases, the metal deficit may be due to the unaccounted factor of duality. An example is the double system $\pi \mathrm{Sgr}$, consisting of two giants of class $\mathrm{F}$, which, apparently, have a solar chemical composition. However, if we study $\pi \mathrm{Sgr}$ without regard for duality, a fictitious deficit of many elements is observed. Attempts were made to find stars belonging to the early subclasses A, having narrow spectral lines, and which would have a solar chemical composition. But all the stars studied had any deviations from the normal composition, hence the conclusion was made that near the subclass A0, normal stars are extremely rare.

There is an assumption that normal A-stars belonging to the main sequence, with slow rotation ( $\mathrm{v}<100 \mathrm{~km} / \mathrm{s})$, mostly evolve in Am. Therefore, it is unlikely to find a star with a solar chemical composition among them. However, at a rotation speed exceeding $100 \mathrm{~km} / \mathrm{s}$ due to meridional circulation (stirring) caused by rotation, chemical anomalies can not be retained in the atmosphere. It is logical to assume that among fast-rotating A-stars, many should have a normal chemical composition. Unfortunately, it is rather difficult to investigate such objects, since the lines in their spectra are very wide due to rotation. It is especially difficult to determine the content of heavy and rare-earth elements, since their lines are weak and few. The most favourable option, when the speed of rotation is large, but the star we see from the pole. However, this situation is extremely rare. [5]

Am-stars.

They are stars with a high content of heavy elements. This group, quite numerous, is sometimes called "metallic" (metallic-line stars) stars. They have an effective temperature ranging from 7300 to $10000 \mathrm{~K}$. And $\log \mathrm{g}$ from 3.5 to 4.5 (belong to the classes of luminosity V-IV). All Am-stars are near the main sequence, that is, when leaving it, the chemical anomalies somehow disappear. The chemical composition of elements of the iron group is usually close to solar, or slightly elevated. In general, there is a reduced or normal content of light elements and an increasing excess of heavy metals as the ordinal number increases. At a speed of rotation at the equator greater than $100 \mathrm{~km} / \mathrm{s}$ (this is the limiting speed), chemical anomalies disappear. In a number of cases Am stars are double, and this cannot be ignored, since the "visible" chemical composition of a binary system, determined without regard for duality, is far from the real chemical composition of the components. Thus, in order to identify real deviations, it is necessary to study the composition of each component of the binary star separately.

For Am stars, there is no noticeable magnetic field, and besides, they have no noticeable variations in the spectral lines that could tell us about the presence of in homogeneities in the chemical composition on the surface of stars.

Ap-stars.

These stars detected magnetic fields reaching $30 \mathrm{kG}$. At the same time, Ap-stars show significant anomalies in the chemical composition. Ap-stars are usually divided into two large subgroups-SrCrEu and Si. For some objects a refined classification is applied, for example $\mathrm{SrCr}, \mathrm{SrEu}, \mathrm{CrEu}$. The belonging of the star to the $\mathrm{SrCrEu}$ subgroup does not at all mean that all three elements in it differ in anomalous content from other lines in terms of their anomalous contents. The SrCrEu subgroup belongs to the effective temperature range from 7500 to $10000 \mathrm{~K}$. The subgroup Si corresponds to higher temperatures, from 9000 to $16000 \mathrm{~K}$. The values of $\log \mathrm{g}$ correspond to GP stars $(3.8-4.2)$. 
The chemical composition of elements of the iron group is usually close to solar, or elevated. Super-heavy metal lines (Z> 72), such as $\mathrm{W}, \mathrm{Pb}, \mathrm{U}$, are observed in the spectra of Ap stars. But they are few and often blended. In the Si subgroup, because of the high temperatures, the lines of super heavy elements can be observed only with strong excesses of these elements. The magnetic fields of Ap-stars often show periodic changes. With the same period, shine and color change. Also, the lines of some elements in the spectrum change. Magnetic and spectral variability can be explained if we assume the model of an inclined rotator. In this model, the axis of the dipole magnetic field, the axis of rotation of the star, the line of sight do not coincide with each other. Rotation leads to periodic changes in the spectral lines, if it is assumed that on the surface of the star the corresponding elements are concentrated in spots. When the star is visible from the pole of rotation, the magnetic and spectral variations will not be chemically peculiar. [6]

\section{OBSERVATIONS AND PROCESSING OF ECHELLE-SPECTRA}

From the point of view of observations using modern high-resolution echelle spectrometers, stars of the A-F classes with narrow absorption lines (with a slow speed of the projection of axial rotation to the line of sight) are the most optimal objects.[7]

In 2009, a 1.5-m telescope of the RTT-150 Kazan Federal University, established in Turkey, launched a program of observations of a sample of A-F stars in a joint research program with Prof. S. Adelman (USA) and his Turkish counterparts. At the first stage, the stars included in the list of observations proposed for the observations of Prof. S. Adelman on the basis of the experience of his previous studies of A-F stars. It was been obtained high-resolution echelle spectra $(R=50000$, Delta Lambda $=0.15$ A) for a set of 20 stars using Andor's large-format CCD $(2048 \times 2048$ pixels) mounted on a Coute-echelle RTT-150 spectrometer. I was asked to perform a preliminary study of the chemical composition of the 12 stars of this program (with narrow absorption lines) by means of processing and analysis of spectra by the method of atmospheric models available at the Department of Astronomy and Space Geodesy of KFU. The list of these stars is given in Table 3.1. [8]

Table 3.1. The main parameters of the stars from the SIMBAD database and the number of spectral lines whose equivalent widths were measured in this paper in the spectrum of the corresponding star.

\begin{tabular}{|l|l|l|l|l|}
\hline HD number & Star name & V & Sp & N lines \\
\hline 165908 & 99 Her & 5.07 & F7 V & 570 \\
\hline 112735 & - & 7.28 & G0 IV-V & 470 \\
\hline 130044 & - & 6.74 & F0 IV-V & 308 \\
\hline 157329 & - & 6.91 & F8 IV-V & 213 \\
\hline 32537 & 9 Aur & 4.98 & F0 IV-V & 85 \\
\hline 26322 & 44 Tau & 5.40 & F2 IV-V & 694 \\
\hline 147135 & - & 6.32 & F0 III-IV & 140 \\
\hline 214994 & Omi Peg & 4.80 & A1 IV & 191 \\
\hline 95608 & 60 Leo & 4.41 & A1m IV-V & 95 \\
\hline 187340 & - & 5.91 & A2 III & 131 \\
\hline 72037 & 2 UMa & 5.46 & A2m IV-V & 267 \\
\hline 195295 & 41 Cyg & 4.02 & F5 I-II & 408 \\
\hline
\end{tabular}

In general, the equivalent widths of 3500 spectral lines in the echelle spectra of these 12 stars were measured. The processing of the echelle spectra was performed using a modified version of the DECH20 program by GA Galazutdinova.

\section{DETERMINATION OF THE FUNDAMENTAL PARAMETERS OF STARS}

To calculate the contents of chemical elements, it is necessary to have an atmosphere model with predetermined parameters-the effective temperature Teff, gravity acceleration at the surface logg, the total metal content (relative to the solar one).

1. Determination of the effective temperature Teff.

In this paper, the effective temperature was determined by the color indices B2 - G, B2 - V1, b - y, B - V, U - B, which were calibrated in the literature in the infrared flow scale. The corresponding equations for the relationship between temperature and color indices were taken from the following articles: 


\section{International Advanced Research Journal in Science, Engineering and Technology}

Vol. 6, Issue 3, March 2019

The equations of the relationship between the temperature and the color indices of the Geneva medium-band system: 1)

$$
\begin{array}{lrl}
\theta_{\text {eff }}=1.626(B 2-G)+1.665, & -0.825 \leq B 2-G<-0.725, \\
\theta_{\text {eff }}=0.81(B 2-G)+1.07, & -0.725 \leq B 2-G<-0.640, \\
\theta_{\text {eff }}=0.458(B 2-G)+0.835, & B 2-G \geq-0.640 .
\end{array}
$$

2)

$$
\begin{gathered}
\theta_{\text {eff }}=2.306(B 2-V 1)+0.917 \\
\quad \text { for }-0.300 \leq B 2-V 1 \leq-0.160 \\
\theta_{\text {eff }}=0.640(B 2-V 1)+0.632 \\
\text { for }-0.160 \leq B 2-V 1 \leq+0.730
\end{gathered}
$$

3) $\theta$ eff $=0.629+0.644 *(\mathrm{~B} 2-\mathrm{V} 1)+0.065 *(\mathrm{~B} 2-\mathrm{V} 1) * * 2$

4) The form of equation $T=A+B * p+C^{*}\left(p^{\wedge} 2\right)$, where $p$ is the color index

5) $\theta$ eff $=(\mathrm{U}-\mathrm{B})-0.72 *(\mathrm{~B}-\mathrm{V})-0.05\left((\mathrm{~B}-\mathrm{V})^{\wedge} 2\right)$

6) $\theta$ eff $=0.838+0.501 *(B 2-G)-0.030 *\left((B 2-G)^{\wedge} 2\right)$

In the SYMBAD database I found the values of the above color indicators and determined the effective temperature

\begin{tabular}{|c|c|c|c|}
\hline 99Her Equation & color index & Color index value & Teff \\
\hline 1. $\theta=0.458 *(B 2-G)+0.835$ & B2-G & -0.013 & 6080 \\
\hline 2. $\theta=0.640 *(\mathrm{~B} 2-\mathrm{V} 1)+0.632$ & B2-V1 & 0.304 & 6094 \\
\hline $\begin{array}{l}\text { 4. } T=A+B * p+C^{*}\left(p^{\wedge} 2\right) \\
\text { a) } A=7995 B=-7246 C=3284\end{array}$ & B2-V1 & 0.304 & 6096 \\
\hline b) $\mathrm{A}=9865 \mathrm{~B}=-13319 \mathrm{C}=7483$ & $\mathrm{~b}-\mathrm{y}$ & 0.356 & 6072 \\
\hline c) $\mathrm{A}=8515 \mathrm{~B}=-5270 \mathrm{C}=1440$ & $\mathrm{~B}-\mathrm{V}$ & 0.475 & 6337 \\
\hline d) $\mathrm{A}=7749 \mathrm{~B}=-7911 \mathrm{C}=7856$ & B2-V1 & 0.304 & 6070 \\
\hline e) $\mathrm{A}=9034 \mathrm{~B}=-12562 \mathrm{C}=11813$ & $\mathrm{~b}-\mathrm{y}$ & 0.356 & 6060 \\
\hline f) $\mathrm{A}=9134 \mathrm{~B}=-8600 \mathrm{C}=5398$ & $\mathrm{~B}-\mathrm{V}$ & 0.475 & 6267 \\
\hline 3. $\theta=0.629+0.644 *(\mathrm{~B} 2-\mathrm{V} 1)+0.065 *\left((\mathrm{~B} 2-\mathrm{V} 1)^{\wedge} 2\right)$ & B2-V1 & 0.304 & 6065 \\
\hline 5. $\theta=(\mathrm{U}-\mathrm{B})-0.72 *(\mathrm{~B}-\mathrm{V})-0.05 *\left((\mathrm{~B}-\mathrm{V})^{\wedge} 2\right)$ & $(\mathrm{U}-\mathrm{B}),(\mathrm{B}-\mathrm{V})$ & $1.207,0.475$ & 6154 \\
\hline 6. $\theta=0.838+0.501 *(\mathrm{~B} 2-\mathrm{G})-0.030 *\left((\mathrm{~B} 2-\mathrm{G})^{\wedge} 2\right)$ & B2-G & -0.013 & 6065 \\
\hline
\end{tabular}
values.

Below are examples of determining the effective temperature for some stars by different color indices and by calibrations of different authors. The most deviant values were discarded. The resulting accepted (average) values of Teff obtained for the investigated stars are given in Table 1. On average, the error in determining the effective value is $100 \mathrm{~K}$.

Table 1

2. Determining the value of $\log \mathrm{g}$.

The determination of the acceleration due to gravity in this work was carried out taking into account the fact that for all stars there are high-precision values of trigonometric parallaxes measured by the satellite GIPPARCOS. Knowing the effective temperature and luminosity of the star, we can determine $\log g$ by the formula: $\log g=4 * \log ($ Teff $)+0.4 *$ $\mathrm{Mv}+\log (\mathrm{M} / \mathrm{Mo})-12.51$,

where the constant value 12.51 reflects the solar values Teff $=5770 \mathrm{~K}$,

$\log \mathrm{g}=4.44, \mathrm{Mv}=\mathrm{V}+5+5 * \log \pi$

The trigonometric parallax values were taken from the SYMBAD database

Table 2

\begin{tabular}{|l|l|l|l|l|}
\hline Star & $\mathrm{V}$ & $\pi, \operatorname{arcsec}$ & $\mathrm{Mv}$ & $\log \mathrm{g}$ \\
\hline 99 Her & 5.07 & $0.064+/-0.0005$ & 4.09 & 4.4 \\
\hline
\end{tabular}




\title{
International Advanced Research Journal in Science, Engineering and Technology
}

\author{
Vol. 6, Issue 3, March 2019
}

\section{CONCLUSION}

Within the framework of this work, echelle spectra of star 99Her of spectral classes A-F has been proceed, obtained with a 1.5-meter telescope PTT-150. As a result of processing the spectra, the equivalent widths of unbend and weakly blended absorption lines were measured, 350 lines this star. The measured equivalent line widths can be used for other studies of the atmospheres of the A-F stars. For 99Her star, the fundamental parameters of atmospheres-the effective temperature, the acceleration of gravity, the microturbulent velocity, were determined, and the content of the chemical elements was calculated (on average for 10-20 chemical elements for each star. Comparison with the published calculations of other authors has shown the reliability of the results obtained. Within the accuracy of the determination of the chemical composition, our results agree with those published. Stars with temperatures of 8000-9000 K show a significant excess of elements heavier than iron and some deficiency of light elements. These results, apparently, are a consequence of the effects of light pressure and gravitational diffusion of atoms and ions in atmospheres of calm and radiative atmospheres of A-stars. Unfortunately, the chemical composition of the atmospheres of our star does not reflect the real chemical composition of the substance from which originated

\section{REFERENCES}

[1]. O.V. Abramova, "The Chemical Evolution of Gas in Galaxies." The role of accretion and outflow of gas "edited by D.Sc. Zasova A.V.

[2]. L.I. Mashonkina, V.V. Shimansky, N.A. Sakhibullin "Non-LTE effects in the spectral lines of NaI in the atmospheres of stars of different types", Astronomical Journal, 2000, vol. 77, No. 12, p. 1-16

[3]. S.J. Adelman, I.H. Cay, H.G. Tektunali, A.F. Gulliver, A. Teker «Elemental abundance analyses with DAO spectrograms: XXXI. The early F supergiants $v$ Her (F2 II) and 41 Cyg (F2 Ib-II) ". Astron. Nachr. , 2008, AN 329, No. 1, p. 4- 9.

[4]. S.J. Adelman, H. Caliskan, D. Kocer, I.H. Cay, H.G. Tektunali «Elemental abundance analyses with DAO spectrograms - XXIII. The superficially normal stars 28 And (A7 III) and 99 Her (F7 V), Mon. Not. R. Astron. Soc., 2000, No. 316, p. 514-518

[5]. S.J. Adelman, O.I. Pintado, F. Nieva, K.E. Rayle, S.E. Sanders, Jr. "On the effective temperatures and the surface gravities of the superficially normal main sequence band B and A stars", Astronomy And Astrophysics, 2002, v.392, p.1031-1037

[6]. F. Castelli, R. Kurucz, "New Grids of ATLAS9 Model Atmospheres", IAU Symp. No 210, Modeling of Stellar Atmospheres, eds. N. Piskunov et al. 2003, poster A20

[7]. A.F. Gulliver, S.J. Adelman, T.P. Friesen "A spectroscopic atlas of Pegasi (A1 IV) $\lambda \lambda$ 3826- 4882", Astronomy And Astrophysics, 2004, v.413, p. 285-291

[8]. I.H. Cay "A spectroscopic analysis of the metallic-line star 2 UrsaeMajoris (A2m)", The A-Star Puzzle, Proceedings IAU Simposium, 2004, No. 224, p. 673-682.

[9]. C. Soubiran, P.Girard “Abundance trends in kinematical groups of the Milky Way’s disk”, Astronomy And Astrophysics, 2005, v.438, p.139151

[10]. W. Zima, H. Lehman, Ch. Sturtz, I.V. Ilyin, M. Breger «High-resolution spectroscopy of the $\delta$ Scuti star 44 Tau: photospheric element abundances and mode indenfication», Astronomy And Astrophysics, 2007, v.471, p.237-245

\section{BIOGRAPHY}

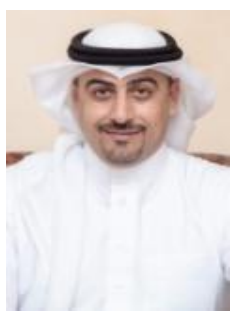

Dr. Omar Alhawi has extensive teaching experience in the department of Science and Math at AMA International University-Bahrain and University of Samrra, most of it focused on Physics and Mathematics. His work provides a useful link between Physics and mathematics in the department, encouraging research and teaching collaborations. He was awarded with $\mathrm{PhD}$ by Kazan Federal University in 2013. His thesis was entitled 'Investigation of the physical parameters and chemical composition of A-F Stars atmospheres'. He has 12 years teaching experience. His research and teaching interests fit extremely well with the requirements of this post and with existing members of staff. 